\title{
ABSORÇÃO DE NUTRIENTES POR MUDAS DE IPÊ-ROXO (Tabebuia impetiginosa (Mart.) Standl.) EM SOLUÇÃO NUTRITIVACONTAMINADAPOR CÁDMIO ${ }^{1}$
}

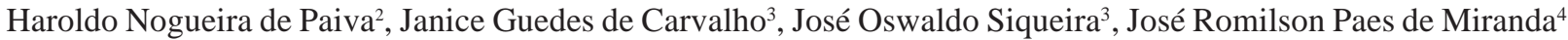
e Antônio Rodrigues Fernandes ${ }^{5}$

\begin{abstract}
RESUMO - Mudas de ipê-roxo, conduzidas em solução nutritiva de Clark, foram submetidas a doses crescentes de Cd: 0, 22, 44, 88 e $132 \mu \mathrm{mol} / \mathrm{l}$, em delineamento de blocos ao acaso, por um período de 60 dias. Foram avaliados os teores e os conteúdos de Cd, de macro e de micronutrientes, na raiz, no caule e nas folhas. Os resultados mostraram que na raiz ocorreu redução no teor dos macronutrientes, enquanto nas folhas não houve efeito significativo. O teor radicular de $\mathrm{Cu}$, Fe e Mn aumentou e o de Zn diminuiu na presença de Cd. O conteúdo radicular de macronutrientes diminuiu com a aplicação de Cd, ao passo que os conteúdos caulinar e foliar não foram afetados. O teor e o conteúdo de Cd nas diferentes partes das mudas foram crescentes em função da dose do metal pesado.
\end{abstract}

Palavras-chave: Tabebuia impetiginosa, ipê-roxo, Cd, metal pesado e nutrição.

\section{NUTRIENTS ABSORPTION BY SEEDLINGS OF IPÊ-ROXO (Tabebuia impetiginosa (Mart.) Standl.) IN NUTRIENT SOLUTION CONTAMINATED BY CADMIUM}

\begin{abstract}
Seedlings of Tabebuia impetiginosa cultivated in Clark nutrient solution were submitted to the following Cd levels: 0, 22, 44, 88 and $132 \mu \mathrm{mol} / \mathrm{l}$, arranged in a randomized block design for 60 days. The plants were picked up and separated into root, stem and leaf, which were analyzed regarding Cd, macro, and micronutrient content and accumulation. The results showed that the macronutrient content of the root was reduced, while that of the leaves did not have a significant effect. The $\mathrm{Cu}, \mathrm{Fe}$, and $\mathrm{Mn}$ contents of the root increased and the $\mathrm{Zn}$ content decreased. Root macronutrient accumulation reduced and leaf accumulation was not affected. The Cd content and accumulation in the different parts of the seedlings increased with the heavy metal dose.
\end{abstract}

Key words: $\quad$ Tabebuia impetiginosa, ipê-roxo, Cd, heavy metal, nutrition.

\section{INTRODUÇÃO}

Por metais pesados entende-se todos aqueles elementos químicos cuja densidade seja superior a $5 \mathrm{~kg} / \mathrm{dm}^{3}$. Dentre estes podem ser citados: cádmio, chumbo, níquel, zinco, cobre e manganês. Alguns dos elementos denominados metais pesados, em pequenas concentrações, são nutrientes essenciais ao desenvolvimento de plantas.
Entretanto, concentrações excessivas de metais, essenciais e não-essenciais, resultam em fitotoxicidade.

A toxicidade de metais pesados é atribuída à sua habilidade de se ligar a enzimas, provocando sua inativação ou mesmo aumentando a atividade de algumas, resultando em alterações na sua função catalítica (Van Assche \& Clijsters, 1990).

1 Recebido para publicação em 17.7.2002 e aceito para publicação em 30.4.2004.

Parte da tese do primeiro autor, apresentada à Universidade Federal de Lavras.

2 Departamento de Engenharia Florestal, Universidade Federal de Viçosa - UFV, 36570-000 Viçosa-MG, <hnpaiva@ufv.br>. ${ }^{3}$ Departamento de Ciência do Solo, Universidade Federal de Lavras - UFLA, 37200-000 Lavras-MG. ${ }^{4}$ Departamento de Engenharia Florestal, Universidade Federal da Paraíba - UFPB, 58700-970 Patos-PB. ${ }^{5}$ Departamento de Solos, Universidade Federal Rural da Amazônia - UFRA, 66077-530 Belém-PA. 
Há evidências na literatura de que as plantas que crescem em solos contaminados por metais pesados não podem prevenir a absorção, mas somente restringi-la, acumulando, assim, estes metais em seus tecidos (Peterson, 1971).

A presença de Cd no meio de crescimento provoca distúrbios nutricionais, sendo esta resposta muito variável entre as espécies. O teor de $\mathrm{K}$, Ca e Mn nas raízes e o de $\mathrm{Ca}, \mathrm{P}, \mathrm{Fe}, \mathrm{Mn}$ e Cu na parte aérea de mudas de Betula pendula diminuíram significativamente pelo tratamento com $2 \mu \mathrm{mol} / \mathrm{l}$ Cd em solução nutritiva (Gussarson et al., 1996). Já em Eucalyptus maculata e Eucalyptus urophylla, ao aplicar doses entre 0 e $180 \mu \mathrm{mol} / \mathrm{l} \mathrm{Cd}$, houve redução no teor de $\mathrm{Cu}, \mathrm{Zn}$, Mn e Fe na parte aérea, com as menores doses de $\mathrm{Cd}$, ao passo que o teor destes nutrientes aumentou com o crescimento das doses. Nas raízes, houve aumento no teor de $\mathrm{Cu}$ e Fe e redução de Zn e Mn (Soares, 1999). O mesmo autor verificou acréscimo no teor de $\mathrm{P}$, tanto na raiz quanto na parte aérea, enquanto os teores de K e de S não foram afetados na parte aérea, mas foram reduzidos no sistema radicular. Os teores de Ca e de Mg na parte aérea tiveram resposta tipo quadrática e raiz-quadrática, respectivamente, e na raiz o Ca teve aumento e o Mg redução, em função das doses de Cd.

Na matéria seca de raiz de mudas de Cedrela fissilis foram observados aumento linear nos teores de P, Fe e $\mathrm{Cu}$, redução no teor de $\mathrm{Zn}$, resposta quadrática positiva sobre o teor de $\mathrm{Mg}$ e $\mathrm{Mn}$, resposta quadrática negativa sobre o teor de $\mathrm{S}$ e nenhum efeito significativo sobre o teor de K e Ca. Nas folhas foram observados aumento linear no teor de Fe, resposta quadrática positiva sobre o teor de $\mathrm{Cu}$ e $\mathrm{Zn}$, resposta quadrática negativa sobre o teor de P, Ca e Mn e nenhum efeito sobre os teores de K, S e Mg com a aplicação de doses entre 0 e $132 \mu \mathrm{mol} / \mathrm{l} \mathrm{Cd}$ (Paiva et al., 2001).

A resposta apresentada pelas espécies quando submetidas a ambientes contaminados por Cd é muito variável, sendo necessário testar o comportamento de cada espécie em face da contaminação. Em ambiente multicontaminado com metais pesados, Marques et al. (2000) verificaram que o ipê-roxo (Tabebuia impetiginosa (Mart.) Standl.) foi uma das espécies menos afetadas pela contaminação, sendo, assim, uma espécie potencial para utilização em solos degradados. No entanto, há a necessidade de verificar a influência desta contaminação sobre a absorção de nutrientes por parte dessa espécie. Diante disto, o objetivo deste trabalho foi verificar o efeito da aplicação de doses crescentes de Cd sobre o teor e o conteúdo de macro e micronutrientes e de Cd em mudas de ipê-roxo, em solução nutritiva.

\section{MATERIALEMÉTODOS}

O experimento foi conduzido em casa de vegetação do Departamento de Ciência do Solo da Universidade Federal de Lavras - UFLA, utilizando-se mudas de ipêroxo (Tabebuia impetiginosa (Mart.) Standl.).

As mudas foram produzidas em substrato que continha areia lavada, e quando apresentavam altura média de $5 \mathrm{~cm}$ ou dois pares de folhas definitivas foram repicadas para bandejas plásticas com capacidade de 35 litros, contendo solução nutritiva de Clark (Clark, 1975). A concentração de todos os nutrientes foi reduzida a $30 \%$, com aeração constante. Neste ambiente, as mudas permaneceram por 15 dias, quando a solução foi substituída e a concentração de todos os nutrientes foi elevada para 50\% da normal. Ao final de 15 dias, as mudas foram individualizadas em vasos plásticos, com capacidade de $900 \mathrm{ml}$, contendo solução nutritiva com 100\% da força iônica, sendo trocada a cada dez dias.

Após 40 dias, foram aplicadas doses crescentes de cádmio. Estas consistiram de 0, 22, 44, 88 e 132 mol/l, usando-se como fonte o cloreto de cádmio $\left[\mathrm{CdCl}_{2}\right.$. 2,5 $\mathrm{H}_{2} \mathrm{O}$ ]. Na preparação de todas as soluções-estoque dos nutrientes e do cádmio, empregaram-se reagentes PA. A solução nutritiva foi preparada com água deionizada, e durante o intervalo de renovação da solução o volume dos vasos foi completado, sempre que necessário, utilizando-se também água deionizada.

As plantas foram mantidas por 60 dias em exposição ao metal pesado, com renovação da solução nutritiva a cada dez dias, mantendo-se o pH em 5,5 pela adição de $\mathrm{NaOH}$ ou $\mathrm{HCl}$ 0,1 mol/l. Após este período procedeu-se à colheita das plantas, separando-as em raiz, caule e folhas.

As raízes, o caule e as folhas foram lavados em água destilada e secos em estufa com circulação de ar a $65^{\circ} \mathrm{C}$, até peso constante. Determinou-se, em balança de precisão (0,01 g), o peso de matéria seca, que em seguida foi moída em moinho tipo Wiley, equipado com peneira de $0,38 \mathrm{~mm}$, para ser analisada quimicamente.

Após a digestão nítrico-perclórica, os teores de Ca, $\mathrm{Mg}, \mathrm{Fe}, \mathrm{Cu}, \mathrm{Mn}, \mathrm{Zn}$ e Cd na raiz, no caule e nas folhas foram determinados por espectrofotometria de absorção atômica. Os teores de $\mathrm{P}$ foram determinados por 
colorimetria, os de S por turbidimetria e os de K por fotometria de chama (Malavolta et al., 1997).

Os conteúdos de P, K, S, Ca, Mg, Fe, Cu, Mn, Zn e $\mathrm{Cd}$ na raiz, no caule, nas folhas, na parte aérea e total foram calculados com base nos teores e nas produções de matéria seca.

O delineamento estatístico adotado foi o de blocos ao acaso, com cinco tratamentos e cinco repetições, sendo cada repetição representada por um vaso com uma planta, perfazendo, assim, um total de 25 plantas.

Os dados foram submetidos à análise de variância, e foram ajustadas equações de regressão entre as doses de cádmio aplicadas e o teor e o conteúdo dos diferentes elementos analisados.

\section{RESULTADOS E DISCUSSÃO}

Os teores radicular e caulinar de macronutrientes foram afetados de forma significativa pela presença de cádmio em solução nutritiva, ao passo que o teor foliar destes nutrientes não o foi (Quadro 1). O teor de P na matéria seca de raiz diminui de forma linear com a aplicação de Cd. No entanto, até a maior dose aplicada
(132 $\mu \mathrm{mol} / \mathrm{l} \mathrm{Cd}$ ) não atingiu grau de deficiência, pois de acordo com Bergmann (1992) teores de P entre 1 e 5 g/kg são requeridos para o ótimo crescimento das plantas, e, no presente estudo, este teor alcançou 1,9 g/kg.

Redução no teor radicular de P também foi observada por Gussarson (1994) em Betula pendula, em resposta à aplicação de Cd, em solução nutritiva. Estes resultados concordam com a afirmação de Kabata-Pendias \& Pendias (1984) de que a presença de metais pesados, como o cádmio, provoca antagonismo na absorção de P.

Na matéria seca de raiz de mudas de ipê-roxo, os teores de K, S, Ca e Mg apresentaram resposta quadrática, tendo os teores de K, Ca e Mg alcançado um mínimo quando da aplicação de 105, 114 e 114 mmol/l Cd, respectivamente, passando a ser crescentes a partir destas doses, podendo este fato ser justificado pelo efeito concentração, uma vez que ocorreu redução na produção de matéria seca de raízes, conforme observado por Paiva et al. (2000b). Neste ponto de mínimo, os teores de K, Ca e Mg foram de 0,99, 0,41 e 0,42 mg/kg, respectivamente, representando um valor 8,15, 4,40 e 1,44 vezes menor que o apresentado pelo tratamento-controle. O teor de $\mathrm{S}$ aumentou com as doses de Cd, alcançando um máximo quando da aplicação de 83,5 $\mu \mathrm{mol} / \mathrm{l} \mathrm{Cd}$, decrescendo desta dose em diante.

Quadro 1 - Equações de regressão para os teores de macronutrientes na raiz, no caule e nas folhas de mudas de ipê-roxo, em resposta a doses crescentes de cádmio ( 0 a $132 \mu \mathrm{mol} / \mathrm{l} \mathrm{Cd})$

Table 1 - Regression equations of macronutrients content in the root, stem and leaves of ipê-roxo seedlings, in response to increasing levels of cadmium (0 to $132 \mu \mathrm{mol} / \mathrm{l} \mathrm{Cd}$ )

\begin{tabular}{|c|c|c|c|}
\hline Nutriente & Parte da Planta & Equação de Regressão & $\mathrm{R}^{2}$ \\
\hline \multirow{3}{*}{$\mathrm{P}$} & Raiz & $\mathrm{Y}=2,6-0,0022 * * \mathrm{X}$ & 0,76 \\
\hline & Caule & $Y=1,6+0,0378^{* *} \mathrm{X}-0,000233^{* *} \mathrm{X}^{2}$ & 0,88 \\
\hline & Folha & n.s. & \\
\hline \multirow{3}{*}{$\mathrm{K}$} & Raiz & $\mathrm{Y}=8,1-0,1353^{* *} \mathrm{X}+0,000644^{* *} \mathrm{X}^{2}$ & 0,90 \\
\hline & Caule & $\mathrm{Y}=8,1+0,0472 * * \mathrm{X}$ & 0,99 \\
\hline & Folha & n.s. & \\
\hline \multirow{3}{*}{$\mathrm{S}$} & Raiz & $\mathrm{Y}=2,5+0,0396 * * \mathrm{X}-0,000237 * * \mathrm{X}^{2}$ & 0,81 \\
\hline & Caule & s.a. $\quad$ l & \\
\hline & Folha & n.s. & \\
\hline \multirow{3}{*}{$\mathrm{Ca}$} & Raiz & $\mathrm{Y}=1,8-0,0244 * * \mathrm{X}+0,000107 * * \mathrm{X}^{2}$ & 0,95 \\
\hline & Caule & $\mathrm{Y}=7,1+0,0123^{* *} \mathrm{X}$ & 0,86 \\
\hline & Folha & n.s. & \\
\hline \multirow{3}{*}{$\mathrm{Mg}$} & Raiz & $\mathrm{Y}=0,6-0,0032 * * \mathrm{X}+0,000014 * * \mathrm{X}^{2}$ & 0,89 \\
\hline & Caule & $Y=1,6+0,0034 * * X$ & 0,71 \\
\hline & Folha & n.s. & \\
\hline
\end{tabular}

** significativo a $1 \%$ de probabilidade, pelo teste F; n.s. não-significativo a 5\% de probabilidade, pelo teste F; e s.a. sem ajuste. 
Essa redução nos teores radiculares de $\mathrm{K}$, Ca e Mg pode, possivelmente, ser explicada pelo efeito de competição entre estes elementos e os cátions divalentes, como o $\mathrm{Cd}^{+2}$ presente em altas concentrações, durante o processo de absorção (Walker et al., 1977; Marschner, 1995), muitas vezes caracterizado como antagonismo (Kabata-Pendias \& Pendias, 1984). No presente trabalho a aplicação de Cd aumentou o teor de S, até determinada dose, embora Kabata-Pendias \& Pendias (1984) tenham relatado que a presença de Cd não afeta a taxa de absorção de S em diferentes espécies vegetais. Como se pode perceber, o conhecimento sobre o comportamento das plantas em face da presença de metais pesados não é semelhante, pois cada espécie apresenta uma resposta quando em ambiente contaminado.

Nas folhas das mudas de ipê-roxo a aplicação de cádmio em solução nutritiva não exerceu efeito significativo sobre o teor de P, K, S, Ca e Mg, fato este que está de acordo com os resultados obtidos por Simon (1998), trabalhando com Helianthus annuus; Lagriffoul et al. (1998), trabalhando com Zea mays; e Soares (1999), trabalhando com mudas de Eucalyptus urophylla e
Eucalyptus maculata. Também em mudas de Cedrela fissilis, Paiva et al. (2001) não observaram efeito significativo da aplicação de Cd sobre o teor foliar de K, S e Mg.

O teor de micronutrientes na raiz e no caule de mudas de ipê-roxo foi afetado de forma significativa pelas doses de Cd aplicadas em solução nutritiva, ao passo que nas folhas apenas o teor de Fe foi afetado (Quadro 2).

Nas raízes, o teor de Cu aumentou de forma linear, corroborando a afirmação de Obata \& Umebayashi (1997) de que o Cd induz ao aumento no teor radicular de Cu em diferentes espécies. Resultados semelhantes foram obtidos por Paiva et al. (2001), trabalhando com mudas de Cedrela fissilis, e por Gussarson et al. (1996), trabalhando com Betula pendula. Kabata-Pendias \& Pendias (1984) afirmam que a presença de Cd provoca interação com a absorção de $\mathrm{Cu}$, podendo, em algumas espécies, ser antagônica e, em outras, ser sinergística, tendo, no presente caso, a interação sido sinergística.

Verifica-se, pela equação mostrada no Quadro 2, que o teor radicular de Fe apresenta resposta quadrática,

Quadro 2 - Equações de regressão para os teores de micronutrientes e de cádmio na raiz, no caule e nas folhas de mudas de ipê-roxo, em resposta a doses crescentes de cádmio (0 a $132 \mu \mathrm{mol} / \mathrm{l} \mathrm{Cd})$

Table 2 - Regression equations of micronutrients and cadmium content in the root, stem and leaves of ipê-roxo seedlings, in response to increasing levels of cadmium (0 to $132 \mu \mathrm{mol} / \mathrm{l} \mathrm{Cd}$ )

\begin{tabular}{|c|c|c|c|}
\hline Nutriente & Parte da Planta & Equação de Regressão & $\mathrm{R}^{2}$ \\
\hline \multirow{3}{*}{$\mathrm{Cu}$} & Raiz & $\mathrm{Y}=17,1+0,1325 * * \mathrm{X}$ & 0,85 \\
\hline & Caule & $\mathrm{Y}=4,8-0,0097 * * \mathrm{X}+0,000258 * * \mathrm{X}^{2}$ & 0,97 \\
\hline & Folha & n.s. & \\
\hline \multirow{3}{*}{$\mathrm{Fe}$} & Raiz & $\mathrm{Y}=1097,0+6,8146 * * \mathrm{X}-0,467043 * * \mathrm{X}^{2}$ & 0,99 \\
\hline & Caule & $\mathrm{Y}=51,0-0,4598^{* *} \mathrm{X}+0,004448^{* *} \mathrm{X}^{2}$ & 0,85 \\
\hline & Folha & $Y=135,0+0,2375 * * X$ & 0,94 \\
\hline \multirow{3}{*}{$\mathrm{Mn}$} & Raiz & $\mathrm{Y}=47,1+0,3074 * * \mathrm{X}$ & 0,93 \\
\hline & Caule & $\mathrm{Y}=18,0+0,1185^{* *} \mathrm{X}$ & 0,92 \\
\hline & Folha & n.s. & \\
\hline \multirow{3}{*}{$\mathrm{Zn}$} & Raiz & $Y=73,0-0,2146 * * X$ & 0,87 \\
\hline & Caule & $Y=80,0+0,6859 * * X-0,006146 * * X^{2}$ & 0,60 \\
\hline & Folha & n.s. & \\
\hline \multirow{3}{*}{ Cd } & Raiz & $\mathrm{Y}=65,0+9,2400 * * \mathrm{X}-0,039354 * * \mathrm{X}^{2}$ & 0,85 \\
\hline & Caule & $\mathrm{Y}=75,2+4,6117^{* *} \mathrm{X}$ & 0,95 \\
\hline & Folha & $Y=8,6+1,2600^{* *} X-0,008193 X^{2}$ & 0,83 \\
\hline
\end{tabular}

** significativo a $1 \%$ de probabilidade, pelo teste F; e n.s. não-significativo a $5 \%$ de probabilidade, pelo teste $\mathrm{F}$.

R. Árvore, Viçosa-MG, v.28, n.2, p.189-197, 2004 
demonstrando que até certa dose a presença de Cd exerce efeito sinergístico sobre a absorção de Fe e que a partir daí passa a apresentar efeito antagônico, conforme mencionado por Kabata-Pendias \& Pendias (1984). Em mudas de Cedrela fissilis, a aplicação de Cd levou a um aumento crescente no teor de Fe (Paiva et al., 2001), ao passo que em mudas de Eucalyptus urophylla e Eucalyptus maculata foi observado comportamento semelhante ao encontrado no presente caso (Soares, 1999).

Quanto aos teores de Mn e de Zn na matéria seca de raiz, observa-se comportamento oposto, ou seja, o teor de Mn apresenta aumento linear e o teor de Zn apresenta redução linear com as doses de Cd. De acordo com KabataPendias \& Pendias (1984), a resposta das plantas ao Cd, em relação ao Mn, pode ser antagônica ou sinergística, tendo, no presente caso, a resposta sido sinergística. Quanto ao Zn, em várias espécies de plantas a sua absorção foi reduzida pela presença de Cd (Jalil et al. 1994a; Yang et al., 1996b; Nascimento et al., 1998; Soares, 1999).

No caule das mudas de ipê-roxo, os teores de $\mathrm{Cu}$ e de Fe diminuíram até determinada dose, passando a ser crescentes a partir de então. Obata \& Umebayashi (1997) preconizam que o Cd estimula a absorção de $\mathrm{Cu}$, porém restringe seu transporte para a parte aérea. Quanto ao teor de $\mathrm{Fe}$, a aplicação de outros metais pesados, como o $\mathrm{Ni}$, restringiu seu transporte em várias espécies de plantas (Yang et al., 1996a), o mesmo podendo ter acontecido com o Cd.

O teor de Mn no caule das mudas de ipê-roxo aumentou linearmente, ao passo que o teor de $\mathrm{Zn}$ apresentou resposta quadrática, com um máximo teor quando da aplicação de 55,8 $\mu \mathrm{mol} / \mathrm{l} \mathrm{Cd}$, alcançando 99,1 mg/kg, 23,9\% superior ao tratamento-controle. Em mudas de Cedrela fissilis, o teor caulinar de Zn também apresentou resposta quadrática, com máximo teor quando da aplicação de $89,4 \mu \mathrm{mol} / \mathrm{l} \mathrm{Cd}$, ponto em que o teor caulinar de $\mathrm{Zn}$ foi $118,1 \%$ superior ao apresentado pelo tratamento-controle (Paiva et al., 2001).

Nas folhas das mudas de ipê-roxo, os teores de $\mathrm{Cu}$, Mn e Zn não foram afetados significativamente pelas doses de Cd, mostrando a não-influência da presença do elemento sobre o teor destes nutrientes na folha. Estes nutrientes também fazem parte do grupo dos chamados metais pesados e não apresentaram nem sinergismo nem antagonismo com a presença de Cd. Por outro lado, o teor de Fe aumentou linearmente, mostrando sinergismo.
A aplicação de doses crescentes de Cd, em solução nutritiva, fez com que o teor deste elemento aumentasse na raiz, no caule e nas folhas das mudas de ipê-roxo (Quadro 2). Resposta quadrática foi observada para o teor de Cd na raiz e nas folhas, enquanto resposta linear foi observada para o teor de Cd no caule. O máximo alcançado pelo teor de Cd na raiz foi 607,4 mg/kg, na dose $117,4 \mu \mathrm{mol} / \mathrm{l} \mathrm{Cd}$, $834 \%$ superior ao tratamentocontrole. Este comportamento confirma resultados anteriores de que a absorção de Cd aumenta com a concentração deste elemento no meio de crescimento (Smith \& Brennan, 1984; Oliveira et al., 1994; Jalil et al., 1994b; Soares, 1999; Paiva et al., 2001). O teor de Cd nas folhas das mudas de ipê-roxo alcançou o máximo de $57,0 \mathrm{mg} / \mathrm{kg}$, na dose $76,9 \mu \mathrm{mol} / \mathrm{l} \mathrm{Cd}$, representando aumento de $563 \%$ em relação ao controle.

Esses teores de Cd, na raiz, no caule e nas folhas das mudas, alcançam valores superiores à faixa, de $5 \mathrm{a}$ $30 \mathrm{mg} / \mathrm{kg}$, considerada potencialmente tóxica para plantas (Kabata-Pendias \& Pendias, 1984).

Ao analisar o conteúdo de macronutrientes, nas diferentes partes das mudas de ipê-roxo (Quadro 3), constata-se que os conteúdos radiculares de $\mathrm{P}$ e $\mathrm{K}$ reduziram-se de forma linear com a aplicação de doses de cádmio. Este fato se deve à redução no teor destes elementos na raiz (Quadro 1), associada à redução na produção de matéria seca, conforme observado por Paiva et al. (2000b).

Os conteúdos de S, Ca e Mg também sofreram redução com a presença de Cd na solução nutritiva. O conteúdo de $\mathrm{S}$ diminuiu de forma linear e os conteúdos de Ca e de Mg apresentaram resposta quadrática, com um ponto de mínimo quando da aplicação de 114 e $105 \mu \mathrm{mol} / \mathrm{l} \mathrm{Cd}$, respectivamente.

No caule, na folha e na parte aérea não houve efeito significativo das doses de Cd sobre os conteúdos dos macronutrientes analisados, reflexo de que o aumento no teor destes nutrientes (Quadro 1) compensou a perda na produção de matéria seca, conforme observado por Paiva et al. (2000b).

Redução no conteúdo de macronutrientes foi observada em várias espécies de plantas, quando cultivadas sob doses elevadas de metais pesados. Assim, Palacios et al. (1998) obtiveram redução no conteúdo de P na matéria seca de raiz e folha em plantas de tomate submetidas a doses entre 0 e $510 \mu \mathrm{mol} / \mathrm{l} \mathrm{Ni}$; Boawn \& Rasmussen (1971) observaram redução no contéudo de

R. Árvore, Viçosa-MG, v.28, n.2, p.189-197, 2004 
Quadro 3 - Equações de regressão para o conteúdo de macronutrientes em diferentes partes de mudas de ipê-roxo submetidas a doses crescentes de cádmio ( 0 a $132 \mu \mathrm{mol} / \mathrm{l} \mathrm{Cd})$

Table 3 - Regression equations of macronutrients accumulation in the different parts of the ipê-roxo seedlings, in response to increasing levels of cadmium (0toa $132 \mu \mathrm{mol} / \mathrm{l} \mathrm{Cd}$ )

\begin{tabular}{|c|c|c|c|}
\hline Nutriente & Parte da Planta & Equações de Regressão & $\mathrm{R}^{2}$ \\
\hline \multirow{5}{*}{$\mathrm{P}$} & Raiz & $\mathrm{Y}=1,6-0,0090 * * \mathrm{X}$ & 0,86 \\
\hline & Caule & n.s. & \\
\hline & Folha & n.s. & \\
\hline & Parte Aérea & n.s. & \\
\hline & Total & $\mathrm{Y}=4,7-0,0130^{* *} \mathrm{X}$ & 0,94 \\
\hline \multirow{5}{*}{$\mathrm{K}$} & Raiz & $\mathrm{Y}=5,7-0,1233 * * \mathrm{X}+0,000639 * * \mathrm{X}^{2}$ & 0,93 \\
\hline & Caule & n.s. & \\
\hline & Folha & n.s. & \\
\hline & Parte Aérea & n.s. & \\
\hline & Total & $\mathrm{Y}=31,3-0,0845^{* *} \mathrm{X}$ & 0,89 \\
\hline \multirow{5}{*}{$\mathrm{S}$} & Raiz & $\mathrm{Y}=1,8-0,0064^{* *} \mathrm{X}$ & 0,97 \\
\hline & Caule & n.s. & \\
\hline & Folha & n.s. & \\
\hline & Parte Aérea & n.s. & \\
\hline & Total & n.s. & \\
\hline \multirow{5}{*}{$\mathrm{Ca}$} & Raiz & $\mathrm{Y}=1,8-0,0244 * * \mathrm{X}+0,000107 * * \mathrm{X}^{2}$ & 0,95 \\
\hline & Caule & n.s. & \\
\hline & Folha & n.s. & \\
\hline & Parte Aérea & n.s. & \\
\hline & Total & n.s. & \\
\hline \multirow{5}{*}{$\mathrm{Mg}$} & Raiz & $\mathrm{Y}=0,4-0,0059 * * \mathrm{X}+0,000028 * * \mathrm{X}^{2}$ & 0,96 \\
\hline & Caule & n.s. & \\
\hline & Folha & n.s. & \\
\hline & Parte Aérea & n.s. & \\
\hline & Total & n.s. & \\
\hline
\end{tabular}

** significativo a $1 \%$ de probabilidade, pelo teste $\mathrm{F}$; e n.s. não-significativo a $5 \%$ de probabilidade, pelo teste $\mathrm{F}$.

P com a aplicação de Zn, em várias espécies; Soares (1999) verificou redução no conteúdo de $\mathrm{P}$ e de $\mathrm{K}$ em mudas de Eucalyptus urophylla e Eucalyptus maculata quando da aplicação de doses crescentes de $\mathrm{Cd}$, Pb e Zn; e Paiva et al. (2000a) observaram redução no contéudo de P, K e $\mathrm{S}$ quando da aplicação de doses crescentes de $\mathrm{Cd}$ em mudas de Cedrela fissilis, em solução nutritiva. Comportamento semelhante foi verificado para o conteúdo de Ca e Mg em diferentes espécies de plantas submetidas a metais pesados, como Cd, Ni, Pb e Zn (Vásquez et al., 1989; Huang e Cunningham, 1996; Palacios et al., 1998; Soares, 1999; Paiva et al., 2000a).

Quanto aos conteúdos de micronutrientes, observase no Quadro 4 que houve resposta diferenciada entre as partes das mudas de ipê-roxo. Assim, a presença de Cd afetou significativamente o conteúdo de $\mathrm{Cu}$ na matéria seca de folha, parte aérea e total, reduzindo-o linearmente, refletindo na diminuição de produção de matéria seca, o que concordando com os resultados obtidos por Paiva et al. (2000b). O conteúdo total apresentou redução de 5,67 e 34,03\% ao aplicar a menor e a maior dose de Cd, respectivamente, em relação ao tratamento-controle. Paiva et al. (2000a), trabalhando com mudas de Cedrela fissilis, em solução nutritiva, aplicando as mesmas doses de Cd usadas no presente estudo, observaram redução de 9,6 e $57,8 \%$ ao aplicar a menor e a maior dose de Cd, respectivamente, em relação ao conteúdo de Cu no tratamentocontrole, o que indica que as mudas de ipê-roxo apresentaram menor redução no conteúdo de $\mathrm{Cu}$, quando comparadas com as mudas de Cedrela fissilis. Redução 
no conteúdo de Cu também foi obtida por Soares (1999), ao trabalhar com mudas de Eucalyptus urophylla e Eucalyptus maculata.

Nas raízes de mudas de ipê-roxo houve resposta quadrática à aplicação de $\mathrm{Cd}$ no conteúdo de $\mathrm{Fe}$, com um ponto de máximo quando da aplicação de $88,2 \mu \mathrm{mol} / \mathrm{Cd}$, reduzindo-o a partir desta dose. Em mudas de eucalipto, Soares (1999) chegou à conclusão de que a aplicação de Cd reduz, de modo geral, o conteúdo de $\mathrm{Fe}$, ao passo que Paiva et al. (2000a) observaram aumento linear no conteúdo radicular de Fe, em mudas de cedro. No presente trabalho o conteúdo de Fe nas folhas e no caule das mudas não apresentou resposta significativa, enquanto Paiva et al. (2000a) verificaram resposta quadrática para o conteúdo de Fe nas folhas e no caule de mudas de cedro. Esses resultados mostram que o comportamento das espécies é diferenciado quando da presença de metais pesados, como o $\mathrm{Cd}$.

O manganês não teve seu conteúdo afetado de forma significativa pela aplicação de Cd, mostrando a não-interferência deste metal pesado (Cd) sobre o seu conteúdo. Já para o Zn observa-se que houve influência significativa da presença de Cd sobre os conteúdos radicular, caulinar e total. Na raiz houve resposta quadrática, com um ponto de mínimo quando da aplicação de $107,4 \mu \mathrm{mol} / \mathrm{l} \mathrm{Cd}$, aumentando a partir desta dose. Os conteúdos caulinar e total de Zn diminuíram linearmente, tendo o conteúdo total reduzido 7,14 e 42,86\% com a aplicação da menor e da maior dose de Cd, respectivamente, em relação ao tratamento-controle. Redução no conteúdo de Zn foi observada por Soares (1999), trabalhando com mudas de eucalipto, e por Paiva et al. (2000a), trabalhando com mudas de cedro.

Quadro 4 - Equações de regressão para o conteúdo de micronutrientes e de cádmio em diferentes partes das mudas de ipêroxo submetidas a doses crescentes de cádmio (0 a $132 \mu \mathrm{mol} / \mathrm{l} \mathrm{Cd})$

Table 4 -Regression equations of micronutrients and cadmium accumulation in the different parts of ipê-roxo seedlings, in response to increasing levels of cadmium (0 to $132 \mu \mathrm{mol} / \mathrm{l}$ Cd)

\begin{tabular}{|c|c|c|c|}
\hline Nutriente & Parte da Planta & Equações de Regressão & $\mathrm{R}^{2}$ \\
\hline \multirow{5}{*}{$\mathrm{Cu}$} & Raiz & n.s. & \\
\hline & Caule & n.s. & \\
\hline & Folha & $\mathrm{Y}=5,0-0,0221^{* *} \mathrm{X}$ & 0,85 \\
\hline & Parte Aérea & $Y=7,0-0,0244 * * X$ & 0,76 \\
\hline & Total & $\mathrm{Y}=18,0-0,0464^{* * X}$ & 0,99 \\
\hline \multirow{5}{*}{$\mathrm{Fe}$} & Raiz & $\mathrm{Y}=313,0+17,4200 * * \mathrm{X}-0,0987 * * \mathrm{X}^{2}$ & 0,70 \\
\hline & Caule & n.s. & \\
\hline & Folha & n.s. & \\
\hline & Parte Aérea & 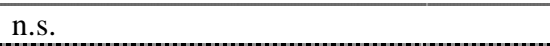 & \\
\hline & Total & $\mathrm{Y}=558,0+18,6700^{* *} \mathrm{X}-0,110294^{* *} \mathrm{X}^{2}$ & 0,73 \\
\hline \multirow{5}{*}{$\mathrm{Mn}$} & Raiz & n.s. & \\
\hline & Caule & n.s. & \\
\hline & Folha & n.s. & \\
\hline & Parte Aérea & n.s. & \\
\hline & Total & n.s. & \\
\hline \multirow{5}{*}{$\mathrm{Zn}$} & Raiz & $\mathrm{Y}=51,0-0,6709 * * \mathrm{X}+0,003124^{* *} \mathrm{X}^{2}$ & 0,95 \\
\hline & Caule & $\mathrm{Y}=46,0-0,2015^{* *} \mathrm{X}$ & 0,83 \\
\hline & Folha & n.s. & \\
\hline & Parte Aérea & n.s. & \\
\hline & Total & $\mathrm{Y}=171,0-0,5552^{* *} \mathrm{X}$ & 0,97 \\
\hline \multirow{5}{*}{$\mathrm{Cd}$} & Raiz & $\mathrm{Y}=8,8+43,7867 * * \mathrm{X}^{0,5}-2,7795 * * \mathrm{X}$ & 0,76 \\
\hline & Caule & $\mathrm{Y}=27,0+2,8700 * * \mathrm{X}-0,0132 * * \mathrm{X}^{2}$ & 0,79 \\
\hline & Folha & $\mathrm{Y}=8,1+1,8300^{* *} \mathrm{X}-0,0123^{* *} \mathrm{X}^{2}$ & 0,92 \\
\hline & Parte Aérea & $\mathrm{Y}=35,0+4,7100 * * \mathrm{X}-0,0256 * * \mathrm{X}^{2}$ & 0,82 \\
\hline & Total & $\mathrm{Y}=14,6+88,4967 * * \mathrm{X}^{0,5}-5,0664^{* *} \mathrm{X}$ & 0,87 \\
\hline
\end{tabular}

** significativo a $1 \%$ de probabilidade, pelo teste F; e n.s. não-significativo a $5 \%$ de probabilidade, pelo teste $\mathrm{F}$. 
O conteúdo de Cd na matéria seca de raiz, caule, folha, parte aérea e total de mudas de ipê-roxo apresentou resposta quadrática para todas as partes das plantas analisadas. O conteúdo máximo atingido nas mudas de ipêroxo foi de 181, 183, 143, 251 e $401 \mu \mathrm{g}$ Cd, significando um aumento de 1.956, 578, 1.665, 617 e 2.646\% em relação ao controle, respectivamente para raiz, caule, folha, parte aérea e total. Nas mudas de ipê-roxo verifica-se que o conteúdo de Cd na parte aérea é superior ao conteúdo na raiz, podendo representar uma menor tolerância desta espécie, pois de acordo com Arduini et al. (1996) a regulação de absorção pela rizosfera, o acúmulo nas raízes, de forma a preservar a integridade e as funções primárias, e o baixo transporte para a parte aérea são considerados os possíveis mecanismos pelos quais o sistema radicular pode contribuir para uma tolerância a metais pesados por espécies arbóreas.

\section{CONCLUSÕES}

Os resultados obtidos permitem concluir que:

- a aplicação de Cd promove redução no teor de P, K, Ca e $\mathrm{Mg}$ na raiz de mudas de ipê-roxo; ao passo que o teor foliar de macronutrientes não é afetado pela presença deste metal pesado;

- o teor radicular de $\mathrm{Cu}, \mathrm{Fe}$ e $\mathrm{Mn}$ aumenta e o de $\mathrm{Zn}$ diminui na presença de $\mathrm{Cd}$, em solução nutritiva

- o teor de Cd na raiz, no caule e na folhas de mudas de ipê-roxo aumenta com as doses aplicadas ( 0 a $132 \mu \mathrm{mol} / \mathrm{lCd}$ );

- o conteúdo radicular de macronutrientes diminui com a aplicação de Cd, ao passo que o conteúdo caulinar e foliar não é afetado significativamente;

- o conteúdo de Mn não é afetado pela aplicação de Cd. O conteúdo total de $\mathrm{Cu}$ e $\mathrm{Zn}$ diminui significativamente, enquanto o conteúdo total de Fe aumenta até $88,2 \mu \mathrm{mol} / \mathrm{lCd}$; e

- o conteúdo de Cd aumentou em todas as partes das plantas analisadas, apresentando resposta quadrática.

\section{REFERÊNCIASBIBLIOGRÁFICAS}

ARDUINI, I.; GODBOLD, D. L.; ONNIS, A. Cadmium and copper uptake and distribution in Mediterranean tree seedlings. Physiologia Plantarum, v. 97, n. 1, p. 111-117, 1996.

R. Árvore, Viçosa-MG, v.28, n.2, p.189-197, 2004
BERGMANN, W. Nutritional disorders of plants: developments, visual and analytical diagnosis. New York: Gustav Fischer Verlang Jena, 1992. 741 p.

BOAWN, L. C.; RASMUSSEN, P. E. Crop response to excessive zinc fertilization of alkaline soil. Agronomy Journal, v. 63, n. 6, p. 874-876, 1971.

CLARK, R. B. Characterization of phosphatase of intact maize roots. Journal of Agriculture and Food Chemistry, v. 23, n. 3, p. 458-460, 1975.

GUSSARSON, M. Cadmium-induced alterations in nutrient composition and growth of Betula pendula seedlings: the significance of fine roots as a primary target for cadmium toxicity. Journal of Plant Nutrition, v. 17, n. 12, p. 21512163, 1994.

GUSSARSON, M. et al. Enhacement of cadmium effects on growth and nutrient composition of birch (Betula pendula) by buthionine sulphoximine (BSO). Journal of

Experimental Botany, v. 47, n. 295, p. 211-215, 1996.

HUANG, J. W.; CUNNINGHAM, S. D. Lead phytoextraction: species variation in lead uptake and translocation. The New Phytologist, v. 134, n. 1, p. 75-74, 1996.

JALIL, A.; SELLES, F.; CLARKE, J. M. Effect of cadmium on growth and the uptake of cadmium and other elements by durum wheat. Journal of Plant Nutrition, v. 17, n. 11, p. 1839-1858, 1994a.

JALIL, A.; SELLES, F.; CLARKE, J. M. Growth and cadmium accumulation in two durum wheat cultivars. Communications in Soil Science and Plant Analysis, v. 25, n. 15/16, p. 2597-2611, 1994b.

KABATA-PENDIAS, A.; PENDIAS, H. Trace elements in soils and plants. Boca Raton: CRC Press, 1984. 315 p.

LAGRIFFOUL, A. et al. Cadmium toxicity effects on growth, mineral and chlorophyll content and activities of stress related enzymes in young maize plants (Zea mays L.) Plant and Soil, v. 200, n. 1, p. 241-250, 1998.

MALAVOLTA, E.; VITTI, G. C.; OLIVEIRA, S. A. Avaliação do estado nutricional das plantas: princípios e aplicações. 2.ed. Piracicaba: POTAFOS, 1997. 319 p.

MARQUES, T. C. L. L. S. M.; MOREIRA, F. M. S.; SIQUEIRA, J. O. Crescimento e teor de metais de mudas de espécies arbóreas cultivadas em solo contaminado com metais pesados. Pequisa Agropecuária Brasileira, v. 35, n. 1, p. 121-132, 2000. 
MARSCHNER, H. Mineral nutrition of higher plants. 2.ed. London: Academic Press, 1995. 889 p.

NASCIMENTO, C. W. A.; FONTES, L. R. F.; NEVES, J. C. L. Mineral composition of two Brazilian corn cultivars as a function of cadmium in the nutrient solution. Journal of Plant Nutrition, v. 21, n. 11, p. 2369-2379, 1998.

OBATA, H.; UMEBAYASHI, M. Effects of cadmium on mineral nutrient concentrations in plants differing tolerance for cadmium. Journal of Plant Nutrition, v. 20, n. 1, p. 97-105, 1997.

OLIVEIRA, J. A. et al. Absorption, accumulation and distribution of cadmium by two soubean cvs. Revista Brasileira de Fisiologia Vegetal, v. 6, n. 2, p. 91-95, 1994.

PAIVA, H. N. et al. Conteúdo de nutrientes em mudas de cedro (Cedrela fissilis Vell.) em resposta a doses crescentes de cádmio. In: SIMPÓSIO NACIONAL RECUPERAÇÃO DE ÁREAS DEGRADADAS, 4., 2000, Blumenau. Anais... Blumenau: SOBRADE/FURB, 2000a. CD Rom.

PAIVA, H. N.; CARVALHO, J. G.; SIQUEIRA, J. O. Efeito de $\mathrm{Cd}, \mathrm{Ni}, \mathrm{Pb}$ e $\mathrm{Zn}$ sobre mudas de cedro (Cedrela fissilis Vell.) e de ipê-roxo (Tabebuia impetiginosa (Mart.) Standley) em solução nutritiva. Revista Árvore, v. 24, n. 4, p. 369-378, 2000b.

PAIVA, H. N.; CARVALHO, J. G.; SIQUEIRA, J. O. Efeito da aplicação de cádmio sobre o teor de nutrientes em mudas de cedro (Cedrela fissilis Vell.). Revista Ciência Florestal, v. 11, n. 2, p. 153-162, 2001.

PALACIOS, G. et al. Effect of nickel concentration on tomato plant nutrition and dry matter yield. Journal of Plant Nutrition, v. 21, n. 10, p. 2179-2191, 1998.
PETERSON, P. J. Unusual accumulations of elements by plants and animals. Scientific Progress, v. 59, p. 505-526, 1971.

SIMON, L. Cadmium accumulation and distribution in sunflower plant. Journal of Plant Nutrition, v. 21, n. 2, p. 341-352, 1998.

SMITH, G. C.; BRENNAN, E. Response of silver maple seedlings to an acute dose of root applied cadmium. Forest Science, v. 30, n. 3, p. 582-586, 1984.

SOARES, C. R. F. S. Toxidez de zinco, cobre, cádmio e chumbo para o eucalipto em solução nutritiva. 1999. 132 f. Dissertação (Mestrado em Solos e Nutrição de Plantas) - Universidade Federal de Lavras, Lavras, 1999.

VAN ASSCHE, F.; CLIJSTERS, H. Effect of metals on enzyme activity in plants. Plant, Cell and Environment, v. 13, p. 195-206, 1990.

VÁSQUEZ, M. D.; POSCHENRIEDER, C.; BARCELÓ, J. Pulvinus structure and leaf abscission in cadmium-treated bean plants (Phaseolus vulgaris). Canadian Journal of Botany, v. 67, n. 9, p. 2756-2764, 1989.

WALKER, W. M.; MILLER, J. E.; HASSETT, J. J. Effect of lead and cadmium upon the calcium, magnesium, potassium, and phosphorus concentration in young corn plants. Soil Science, v. 124, n. 3, p. 145-151, 1977.

YANG, X. et al. Plant tolerance to nickel toxicity: II. Nickel effects on influx and transport de mineral nutrients in four plant species. Journal of Plant Nutrition, v. 19, n. 2, p. 265-279, 1996a.

YANG, X. et al. Cadmium effects on influx and transport of mineral nutrients in plant species. Journal of Plant Nutrition, v. 19, n. 3-4, p. 643-656, 1996 b. 